\title{
Colonoscopy education delivered via the patient portal does not improve adherence to scheduled first-time screening colonoscopy
}

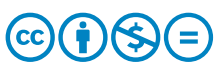

\author{
Authors \\ Cameron $^{2}$ \\ Institutions \\ 1 Department of Gastroenterology and Hepatology, \\ Northwestern University, Chicago, Illinois, United States \\ 2 Division of General Internal Medicine and Geriatrics, \\ Department of Medicine, Northwestern University, \\ Chicago, Illinois, United States \\ 3 Northwestern Memorial Hospital, Chicago, Illinois, \\ United States
}

Rajesh N. Keswani ${ }^{1}$, Dyanna L. Gregory ${ }^{1}$, Mariah Wood ${ }^{1}$, Nancy C. Dolan², Ryan Chmiel ${ }^{3}$, Michael Manka ${ }^{1}$, Kenzie A.

submitted 18.4 .2019

accepted after revision 7.10 .2019

Bibliography

DOI https://doi.org/10.1055/a-1072-4556 |

Endoscopy International Open 2020; 08: E401-E406

(c) Georg Thieme Verlag KG Stuttgart · New York

elSSN 2196-9736

Corresponding author

Rajesh N. Keswani MD MS, 676 N. St. Clair, Suite 1400,

Chicago, IL 60611

raj-keswani@northwestern.edu

\section{ABSTRACT}

Background and study aims Non-adherence to scheduled colonoscopy burdens endoscopic practices and innovative approaches to improve adherence are needed. We aimed to assess the effect of an educational video empha- sizing colonoscopy importance delivered through the electronic health record patient portal upon "no-show" and late cancellation rates (non-adherence) in patients scheduled for first-time screening colonoscopy.

Patients and methods We conducted a single center randomized controlled trial among patients scheduled for their first screening colonoscopy. Patients were randomized to routine care ("control") or video education ("video"). Control patients received a portal message 14 days prior to colonoscopy date; video patients additionally received a link to the educational video.

Results In total, 830 patients ( $59 \%$ female, median age 55 years) were randomized ("control": 406; "video": 424). Nearly all (88\%) opened the message; in the video arm, most $(72 \%)$ watched a majority of the video. Overall, $80 \%$ attended their scheduled colonoscopy appointment (late cancel: $18 \%$, "no show": $1 \%$ ) and $90 \%$ underwent colonoscopy within 3 months of appointment. Adherence rates did not differ between video and control arms for the scheduled appointment (OR 1.2, Cl 0.9-1.8) or for colonoscopy within 3 months of scheduled appointment (OR $1.3, \mathrm{Cl}$ $0.8-2.1$ ). Bowel preparation quality did not differ between the groups.

Conclusion Most patients scheduled for colonoscopy will open a patient portal message and, when delivered, watch an educational video. However, delivery of an educational video two weeks prior to screening colonoscopy appointment did not improve adherence.

\section{Introduction}

Colorectal cancer (CRC) is the second leading cause of cancer death in the United States [1]. Although screening colonoscopy is associated with a reduction in CRC mortality, adherence to screening colonoscopy is suboptimal [2], with less than twothirds of eligible patients undergoing age-appropriate screening. Thus, improving colorectal screening rates is a priority [3]. Among non-adherent patients, some cancel their colonoscopy within days of the procedure ("late cancellation") or never ar- rive for their appointment ("no-show"). These "no-shows" or "late cancellations" result in missed CRC screenings and also waste resources through "empty" endoscopy appointments. Thus, non-adherent patients represent important opportunities to intervene via patient education and subsequently improve both CRC screening rates and operational efficiency.

Numerous CRC screening barriers have been identified, including patient fears, inadequate patient knowledge regarding preparation and screening, limited time for provider counseling, and cultural barriers. Given the time constraints of the pa- 
Patients scheduled for

first-time open-access screening colonoscopy

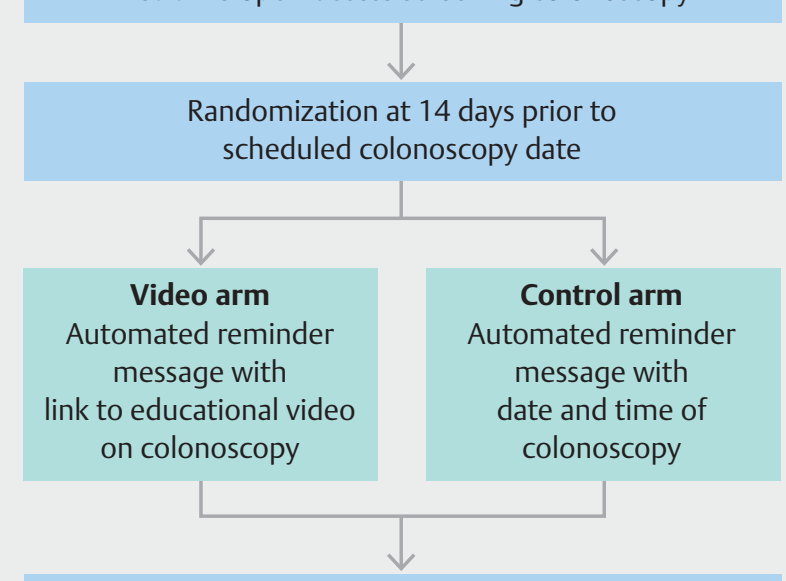

Assess adherence to scheduled screening colonoscopy and bowel preparation quality

- Fig. 1 Study flow diagram.

tient-primary care provider relationship, there is intense interest in effective, patient-centered digital solutions that provide education regarding the importance of CRC screening while addressing common patient concerns about the procedure. However, integrating such education within the clinical workflow is challenging and thus has not been widely implemented. Many studies have focused on commercial products, which add cost to screening colonoscopy. Patient portals, in which secure messages can be sent to patients, are a feature of many electronic health records (EHRs) and present an alternate channel through which to deliver timely, relevant patient education without burdening clinic staff or increasing procedure cost.

We designed a randomized controlled trial (RCT) to assess the impact of a single educational video, delivered automatically through the patient portal, upon adherence to a scheduled screening colonoscopy appointment. The primary aim of this study was to determine if patient education, delivered via the patient portal, improved adherence rates for patients scheduled for their first screening colonoscopy at our institution by reducing "no-show" and "late cancellations" compared to usual care. The secondary aim of this study was to determine the impact of patient education upon bowel preparation quality.

\section{Patients and methods}

\section{Study design}

We conducted a single-blinded RCT of all patients undergoing first-time screening colonoscopy at our institution over a 4month period. Patients were randomized ( $>$ Fig. $\mathbf{1}$ ) by medical record number (odd versus even) to routine care ("control" arm) or routine care with additional delivery of a short educational video through the patient portal ("video" arm). The
Northwestern University Institutional Review Board approved this study and granted a waiver of informed consent.

\section{Study population}

We included all English-speaking patients aged 50 to 75 undergoing their first screening colonoscopy at our institution who also had an activated patient portal account. Only patients scheduled for an "open access" colonoscopy were included in this study. An open access colonoscopy signifies a colonoscopy directly ordered by the primary care provider that occurs without the patient first seeing a gastroenterology provider in clinic and represents the predominant means by which colonoscopies are performed at our institution. Patients previously seen by a gastroenterology physician or advanced practice provider or colonoscopies performed for a diagnostic or surveillance indication were excluded. Pregnant women, prisoners, patients with a medical history of dementia, and patients who were blind and/or deaf were also excluded.

\section{Outcomes}

The primary outcome was scheduled screening colonoscopy adherence, which was assessed in two ways: 1) attending colonoscopy at the initially scheduled date and time and 2) undergoing colonoscopy within 3 months of the scheduled date and time. We identified instances of "late cancellation," which was defined as a cancelled colonoscopy appointment, for any patient-initiated reason, within 2 weeks of scheduled date. Further, a "no-show" was defined as any colonoscopy appointment which was not cancelled but also not attended by the subject. Cancellations due to medical issues and provider schedule changes were excluded. The secondary outcome was bowel preparation quality, as measured by the Boston Bowel Preparation Scale (BBPS) [4]. An adequate BBPS score was defined as a score $\geq 6$.

\section{Intervention}

A multidisciplinary group including a gastroenterologist (RNK), a general internist (NCD) and an expert in patient communication (KAC) created an educational video (https://vimeo.com/ guybauerproductions/review/205963543/6bf79d6cd3) that was designed to ensure patients understood the importance of colorectal cancer while emphasizing the importance of adequate bowel preparation. The video was adapted from previously developed videos encouraging colorectal cancer screening among patients seeking care in internal medicine practices in community-based health centers and academic medical centers [5-8]. The video focused on educating patients regarding the risk of CRC, the effectiveness of screening colonoscopy in preventing CRC, provided an overview of colonoscopy bowel preparation, as well as describing what the patients could expect upon arrival at the clinic.

The intervention strategy was developed based on previous studies using the EHR and the patient portal to provide information to patients prior to a scheduled clinic visit [9]. We implemented the intervention at an academic gastroenterology clinic that uses an institutional electronic health record (Epic, Verona, Wisconsin, United States). Two weeks prior to the 
- Table 1 Study population.

\begin{tabular}{|l|c|c|c|}
\hline & Standard of care (“Control” arm) & Video education (“Video” arm) & \multicolumn{1}{|c|}{$\mathbf{P}$ value } \\
\hline Age & $57.2 \pm 7.0$ & $57.8 \pm 7.3$ & 0.21 \\
\hline Female sex & $239(58.9 \%)$ & $250(59.0 \%)$ & 0.98 \\
\hline Race & & & 0.03 \\
\hline " White & $279(72.7 \%)$ & $269(65.4 \%)$ \\
\hline " African American & $50(13.0 \%)$ & $55(13.4 \%)$ \\
\hline " Other & $55(14.3 \%)$ & $87(21.2 \%)$ \\
\hline
\end{tabular}

scheduled colonoscopy date, all eligible patients received an automated message requesting that they sign in to the secure patient portal system within the EHR, using username and password, to read a new message from their gastroenterologist. Patients in the control arm received a message reminding the patient of their appointment details including time and location. Patients in the video arm received a similar reminder message with additional language asking them to watch a short educational video (195 seconds), along with a custom hyperlink to the video. We tracked whether or not the patient opened the link and, when applicable, how long they watched the educational video.

\section{Statistical analysis}

Prior to study onset, we conducted a sample size calculation. Based on historical data, we anticipated a no-show rate of $10 \%$ for patients undergoing "open access" colonoscopy with a low late cancellation rate. However, we expected our intervention to shift some "no-show" patients to "late cancellation" patients and thus intended to enroll 955 patients, which at an alpha of 0.05 , would provide at least an $80 \%$ power to detect a statistically significant change of $5 \%$ in the no-show/late cancellation rate, allowing for $10 \%$ dropout.

We collected information on the following outcome variables: initial procedure appointment status (as completed, cancellation, late cancellation, no show), 3-month colonoscopy adherence (completed vs. not completed), and adequate BBPS. We also collected the following categorical covariates: gender, race, ethnicity, and MyChart message status (opened vs. unopened); age was collected as a continuous variable. We did not impute missing data. The primary analysis was intent-to-treat and assessed potential association between treatment arm and colonoscopy adherence, as well as "late cancellation" and "no-show" rates. Secondary analyses examined association between treatment arm and adequacy of bowel preparation, assessed semi-continuously as BBPS score and dichotomously as adequate (BBPS $\geq 6$ ) vs. inadequate. We first used univariate analysis using chi-squared or Fisher's exact tests as appropriate for categorical variables, and independent student's t-test for continuous variables. Covariates found to have a significant association at the 0.05 level with both treatment arm and outcome were to be entered into a multiple logistic regression model along with the treatment arm to adjust for potential con- founding. However, as no covariates were significantly associated with both treatment arm and any outcome, univariate test results are reported overall and by treatment arm. As this study was intended to be exploratory and hypothesis generating, adjustments were not made for multiple testing. Analyses were conducted and figures were generated using SAS software, Version 9.4 (Cary, North Carolina, Unite States).

\section{Results}

A total of 945 patients undergoing their first screening colonoscopy at our institution were enrolled and randomized. Of them, 115 were excluded because procedures were rescheduled due to factors outside patient control (e. g. physician schedule, medical hold, insurance). Of the remaining 830, 406 were assigned to the control arm and 424 to the video arm. Mean age was 57.3 years in the control arm and 57.8 years in the video arm $(P=0.30)$, and the majority $(59 \%)$ were female in both groups ( $\triangleright$ Table 1 ).

Nearly all patients ( $n=729,87.8 \%$ ) opened the automated electronic message regarding their colonoscopy. As expected, rates did not differ between control $(349,86.0 \%)$ and video $\operatorname{arm}(380,89.6 \%$, OR 0.7, Cl 0.5, 1.1) subjects.

Among the 424 patients who received the educational video, most ( $n=307,72.4 \%$ ) watched a majority of the video, approximately $17.2 \%$ watched a portion of the video, and the remaining subjects (10.4\%) never opened their electronic message.

\section{Primary outcome: colonoscopy adherence}

Overall, 667 patients (80.4\%) attended their colonoscopy appointment as scheduled. The remaining patients either cancelled their original appointment $(151,18.2 \%)$ or were a "no show" $(12,1.4 \%)$ on the day of their appointment. The rate of adherence ( $>$ Table 2 ) to scheduled colonoscopy did not differ between patients in the video arm $(348,82.1 \%)$ versus control $\operatorname{arm}(319,78.6 \%$; OR 1.2, Cl 0.9-1.8). "No-show" rates also did not differ between the video (5, 1.2\%) and control arms (7, $1.7 \%$; OR 0.7, $\mathrm{Cl} 0.2-2.2$ ) nor did "late cancellation" rates (video: $75,17.7 \%$, control: $87,21.4 \%$; OR $0.8, \mathrm{Cl} 0.6-1.1$ ). We also looked at colonoscopy adherence within 3 months of scheduled colonoscopy date. Overall, 743 patients (89.5\%) underwent colonoscopy within 3 months of scheduled date. The rate of ad- 
- Table2 Colonoscopy adherence rates.

\begin{tabular}{|l|c|c|}
\hline & $\begin{array}{l}\text { Standard of care (“Control” arm) } \\
(\mathbf{N}=\mathbf{4 0 6})\end{array}$ & $\begin{array}{l}\text { Video education (“Video” arm) } \\
\text { (N=424) }\end{array}$ \\
\hline Attended schedulue \\
\hline Cancelled within 2 weeks of scheduled colonoscopy & $319(78.6 \%)$ & $348(82.1 \%)$ \\
\hline “No-show" for colonoscopy & $80(19.7 \%)$ & $71(16.7 \%)$ \\
\hline Attended colonoscopy within 3 months of scheduled date & $358(88.2 \%)$ & $5(1.2 \%)$ \\
\hline
\end{tabular}

herence to colonoscopy did not differ between patients in the video $(385,90.8 \%)$ and control arms $(358,88.2 \%$, OR $1.3, \mathrm{Cl}$ $0.8,2.1)$.

Patients who opened the patient portal message were significantly more likely to adhere to their original colonoscopy appointment (OR 3.6, $\mathrm{Cl} 2.3-5.6$ ) and colonoscopy within 3 months (OR $3.8, \mathrm{Cl} 2.3,6.4$ ) than those who did not open the message. This finding was significant both overall and in subanalyses of both the video and control arms.

Among the 428 patients in the video arm, subjects who opened the message and watched the majority of the educational video ( $\geq 50 \%$ of the video) were more likely to attend scheduled colonoscopy (90.2\%) than those who opened the message but viewed a minority of the video (73.3\%), and those who opened the message but did not watch the video at all $(62.1 \%, P<0.0001)$.

\section{Secondary outcome: bowel preparation quality}

We assessed the impact of the educational video on bowel preparation quality. The rate of bowel preparation adequacy did not differ between the control (99.3\%) and video arms (97.2\%, OR 4.1, Cl 0.9, 19.1). As nearly all patients had a BBPS score $\geq 8$ in both groups ( $91.4 \%$ of the control and $89.2 \%$ of the video arm), we were limited in our ability to detect any improvement in bowel preparation quality. Consistent with this, the overall BBPS score similarly did not differ between the control (mean score $8.7 \pm 0.9$ ) and video arms (mean score $8.7 \pm$ $1.0, P=0.4)$.

\section{Discussion}

In this prospective study of patients undergoing their first screening colonoscopy at our institution, we found that a majority of patients will read an automated pre-colonoscopy message sent through the electronic health record. Further, patients who read this message are more likely to adhere to their screening colonoscopy appointment. However, we did not identify any causal benefit of an added educational video to this message; specifically, the addition of an educational video did not improve colonoscopy adherence rates or bowel preparation quality.

We hypothesized that delivering an educational video, which could be watched at the patient's convenience, would improve colonoscopy adherence rates. Indeed, numerous studies have identified screening reminders or patient-focused interven- tions as increasing patients' use of preventive services, such as colonoscopy [7,10-12], yet few of them have delivered the information to patients via a patient portal [13]. However, in intent-to-treat analyses, we did not identify any improvement in colonoscopy adherence rates with automated delivery of a colonoscopy education video 2 weeks prior to scheduled colonoscopy. Although there are several potential reasons for this outcome, we suspect a major factor is that a message delivered 2 weeks before colonoscopy may be "too late" to alter adherence rates. The major impact of this intervention (delivering a "reminder" message 2 weeks prior to scheduled colonoscopy) appeared to be shifting a historically high "no-show" rate to an increase in "late cancellations." Furthermore, our overall nonadherence rates were higher than anticipated based on historical data, limiting our ability to identify small improvements with our intervention. Finally, patients may need multi-modality reminders at various time points to truly alter behavior [14].

We also assessed the effect of this video upon colonoscopy preparedness, specifically, bowel preparation adequacy. We hypothesized that the video, which emphasized the importance of an optimal bowel preparation, would significantly improve bowel preparation adequacy. However, we similarly did not identify any effect of this educational video upon bowel preparation. This result may reflect a very high baseline bowel preparation adequacy rate, as identified in our sample, presumably a result of our ongoing quality improvement efforts. Furthermore, as the study population was drawn from only those patients who had activated their patient portal accounts, it is possible that we did not reach patients who may be most in need of additional instruction to ensure adequate bowel preparation. Research has demonstrated that more vulnerable patients often experience significant challenges related to the use of patient portals [15-17]; it may be these same patients who are less likely to present with adequate bowel preparation [18]. More recent work has fond that cellular phone text messaging is an effective option at improving colonoscopy adherence [19] and bowel preparation [20]. This low-cost option may be more broadly available to all patients undergoing colonoscopy given the near ubiquitous usage of cellular phones. Similarly, patients with lower health literacy or non-English speakers are at great risk for low-quality bowel preparation, but have lower rates of patient portal usage. Thus, our inclusion of only English speakers likely omitted especially vulnerable populations. The effectiveness of video education targeted to non-English speakers requires further study. 
We did find that patients who opened the electronic message were more likely to adhere to their screening colonoscopy appointment, a relationship seen in both arms. Furthermore, those in the video arm who viewed the majority of the video were more likely to adhere to scheduled screening than those who watched a minority of the video or did not view the video at all. While it is possible that this message encouraged patients to adhere to their scheduled appointment, these results alternatively may reflect the fact that patients who are more engaged (i.e., reading their messages in the patient portal, watching a suggested video to completion) are also more likely to attend their appointments.

There is extensive literature regarding use of manual and automated patient reminders to improve colorectal screening adherence in unscheduled patients. These efforts include patient navigators [21-23], targeted mailings [24], and electronic reminders [13]. However, beyond methods to predict non-adherence $[25,26]$, there is little published literature focused upon reducing colonoscopy late cancellation and "no-show" rates. This area remains important for future exploration as these "empty" colonoscopy appointments both represent a missed opportunity for providing screening to other patients and also are a financial burden. Although the intervention described in this study did not significantly improve adherence rates, the intervention was performed at minimal cost (production of the video) and most patients viewed the video; thus small modifications to this intervention (e.g., video sent at the time of scheduling and/or multiple time points) may be considered to reduce non-adherence rates. Additionally, modifying this intervention so that it does not rely solely upon that patient portal would be of benefit. For example, choosing from a group of interventions including patient portal messages (electronic health record), patient navigators (telephone), and text messaging (cellular phone), targeted to the individual patient based on medical and demographic factors, may be most effective at improving adherence rates.

There are several limitations of this study. This study took place at a single urban academic medical center with high baseline rates of screening colonoscopy adherence. It is possible that this intervention would be more successful in centers with lower baseline adherence rates. Furthermore, it is unclear whether the educational video had an impact on any additional relevant outcomes, including patient knowledge, anxiety, and patient satisfaction as has been seen with other pre-colonoscopy education interventions [27]. The "no-show" rate was notably lower than anticipated based on historical data while rates of procedure rescheduling due to factors beyond patient control were higher than expected. In addition, rates of "late cancellation" were higher than anticipated; we suspect that this occurred, in part, due to the fact that both groups received reminders regarding their procedures and thus may have prompted patients who did not intend to have their procedure to cancel (shifting "no-show" to "late cancellations"). These factors in combination led to the study being underpowered in comparison to original calculations and repeating a similar study with a higher enrollment could provide more definitive results with regard to the negative outcomes seen here.

\section{Conclusion}

In summary, we found that a majority of patients will view a screening colonoscopy educational video delivered via the EHR. However, the addition of this video has no causal impact upon adherence to a scheduled screening colonoscopy appointment or bowel preparation quality. Whether delivery of this low-cost education can improve other relevant outcomes or is useful in alternative settings (such as patients who have not scheduled a screening colonoscopy) remains unclear.

\section{Acknowledgements}

This study was funded by the Northwestern University Digestive Health Foundation. Research reported in this publication was supported, in part, by the National Institutes of Health's National Center for Advancing Translational Sciences, Grant Number UL1TR001422.

\section{Competing interests}

Keswani - Boston Scientific, Consultant (Not Relevant to Subject Matter)

References

[1] Siegel RL, Miller KD, Jemal A. Cancer statistics, 2016. CA Cancer J Clin 2016; 66: 7-30

[2] Liang PS, Wheat CL, Abhat A et al. Adherence to competing strategies for colorectal cancer screening over 3 years. Am J Gastroenterol 2016; 111: 105-114

[3] Karlitz JJ, Oliphant AB, Greenwald DA et al. The American College of Gastroenterology and the $80 \%$ by 2018 colorectal cancer initiative: a multifaceted approach to maximize screening rates. Am J Gastroenterol 2017; 112: 1360-1362

[4] Lai E], Calderwood AH, Doros G et al. The Boston bowel preparation scale: a valid and reliable instrument for colonoscopy-oriented research. Gastrointest Endosc 2009; 69: 620-625

[5] Cameron KA, Francis L, Wolf MS et al. Investigating Hispanic/Latino perceptions about colorectal cancer screening: a community-based approach to effective message design. Patient Educ Couns 2007; 68: 145-152

[6] Makoul G, Cameron KA, Baker DW et al. A multimedia patient education program on colorectal cancer screening increases knowledge and willingness to consider screening among Hispanic/Latino patients. Patient Educ Couns 2009; 76: 220-226

[7] Cameron KA, Persell SD, Brown T et al. Patient outreach to promote colorectal cancer screening among patients with an expired order for colonoscopy: a randomized controlled trial. Arch Intern Med 2011; 171: 642-646

[8] Dolan NC, Ramirez-Zohfeld V, Rademaker AW et al. The effectiveness of a physician-only and physician-patient intervention on colorectal cancer screening discussions between providers and African American and Latino patients. J Gen Intern Med 2015; 30: 1780-1787

[9] Brown T, Goldman SN, Persell SD et al. Development and evaluation of a patient education video promoting pneumococcal vaccination. Patient Educ Couns 2017; 100: 1024-1027 
[10] Fortuna R], Idris A, Winters P et al. Get screened: a randomized trial of the incremental benefits of reminders, recall, and outreach on cancer screening. J Gen Intern Med 2014; 29: 90-97

[11] Sequist TD, Zaslavsky AM, Marshall R et al. Patient and physician reminders to promote colorectal cancer screening: a randomized controlled trial. Arch Intern Med 2009; 169: 364-371

[12] Senore C, Inadomi J, Segnan N et al. Optimising colorectal cancer screening acceptance: a review. Gut 2015; 64: 1158-1177

[13] Sequist TD, Zaslavsky AM, Colditz GA et al. Electronic patient messages to promote colorectal cancer screening: a randomized controlled trial. Arch Intern Med 2011; 171: 636-641

[14] Baker DW, Brown T, Buchanan DR et al. Comparative effectiveness of a multifaceted intervention to improve adherence to annual colorectal cancer screening in community health centers: a randomized clinical trial. JAMA Intern Med 2014; 174: 1235-1241

[15] Tieu L, Schillinger D, Sarkar U et al. Online patient websites for electronic health record access among vulnerable populations: portals to nowhere? J Am Med Inform Assoc 2017; 24: e47-e54

[16] Taha J, Sharit J, Czaja SJ. The impact of numeracy ability and technology skills on older adults' performance of health management tasks using a patient portal. J Appl Gerontol 2014; 33: 416-436

[17] Lyles CR, Fruchterman J, Youdelman M et al. Legal, practical, and ethical considerations for making online patient portals accessible for all. Am J Public Health 2017; 107: 1608-1611

[18] Serper M, Gawron AJ, Smith SG et al. Patient factors that affect quality of colonoscopy preparation. Clin Gastroenterol Hepatol 2014; 12: 451-457

[19] Wu Y, Liang Y, Zhou Q et al. Effectiveness of a short message service intervention to motivate people with positive results in preliminary colorectal cancer screening to undergo colonoscopy: A randomized controlled trial. Cancer 2019: doi:10.1002/cncr.32043

[20] Walter B, Klare P, Strehle K et al. Improving the quality and acceptance of colonoscopy preparation by reinforced patient education with short message service: results from a randomized, multicenter study (PERICLES-II). Gastrointest Endosc 2019; 89: 506-513 e504

[21] Ritvo PG, Myers RE, Paszat LF et al. Personal navigation increases colorectal cancer screening uptake. Cancer Epidemiol Biomarkers Prev 2015; 24: 506-511

[22] Chen LA, Santos S, Jandorf L et al. A program to enhance completion of screening colonoscopy among urban minorities. Clin Gastroenterol Hepatol 2008; 6: 443-450

[23] DeGroff A, Schroy PC 3rd, Morrissey KG et al. Patient navigation for colonoscopy completion: results of an RCT. Am J Prev Med 2017; 53 : 363-372

[24] Green BB, Wang CY, Anderson ML et al. An automated intervention with stepped increases in support to increase uptake of colorectal cancer screening: a randomized trial. Ann Intern Med 2013; 158: 301-311

[25] Blumenthal DM, Singal G, Mangla SS et al. Predicting non-adherence with outpatient colonoscopy using a novel electronic tool that measures prior non-adherence. J Gen Intern Med 2015; 30: 724-731

[26] Partin MR, Gravely A, Gellad ZF et al. Factors associated with missed and cancelled colonoscopy appointments at Veterans Health Administration facilities. Clin Gastroenterol Hepatol 2016; 14: 259-267

[27] Parker S, Zipursky J, Ma $\mathrm{H}$ et al. A web-based multimedia program before colonoscopy increased knowledge and decreased anxiety, sedation requirement, and procedure time. J Clin Gastroenterol 2018; 52: $519-523$ 